\title{
Is Prescribing Custom Orthotics Enough to Prevent and Treat Musculoskeletal Injuries and Degeneration? What if we Integrated Biomechanical $X$-ray Measurements with the Laser Foot Scan Procedure? Could this Reduce Injuries Even More?
}

\author{
Tim Maggs ${ }^{1}$, Steven Brownstein ${ }^{2 *}$ and Alexandros Siozos ${ }^{3}$ \\ ${ }^{1}$ DC, Director, Maggs Sports Chiropractic Biomechanics and Wellness Center Director, Sports Injuries and Biomechanics, USA \\ ${ }^{2}$ MD, Radiologist, Medical Director of Dynamic Medical Imaging, Medical Director of Spinal Kinetics, LLC Union, USA \\ ${ }^{3} \mathrm{MD}$, Consultant in Orthopaedic Surgery, Private Practice, Greece
}

Submission: February 19, 2021; Published: March 02, 2021

*Corresponding author: Steven Brownstein, MD, Radiologist, Medical Director of Dynamic Medical Imaging, Medical Director of Spinal Kinetics, LLC Union, USA

\begin{abstract}
Orthotics and custom-made insoles have been used for decades for both professional and recreational athletes but are not always associated with a successful outcome. In fact, there are occasions where they are associated with worsening of pain and ailments in the back and pelvis area. Our goal is to explain why, at times, orthotics fail to tackle unevenly distributed weight -and load- bearing and to introduce a new measurement method that could help all healthcare professionals to identify, classify and finally treat their patients in a biomechanically optimal manner. To do so, we performed a 3D digital laser foot scan in each of the 351 individuals in our study and ordered the custom made orthotic accordingly. Then, two standing A-P Lumbo-Sacral digital x-rays were obtained, the first with the patient being barefoot and the second while wearing the custom orthotics on the inside of tied shoes. Our results showed a significant alteration in femoral head height difference (fhhd) which was consistent with each patient's clinical findings. That sequence also helped us, in the first of three studies, to identify five different biomechanically flawed patterns and to conclude that each patient falls into one of these five categories. In this $2^{\text {nd }}$ study, our goal is to focus on what percentage of the population falls into the "normal" category of fhhd, which is $3 \mathrm{~mm}$ and below, and what percentage of the population falls into the "abnormal" category, which is greater than $3 \mathrm{~mm}$. The data will show both barefoot and with custom orthotic results. Should these tests be applied to every individual, it might dramatically prevent and reduce the incidence of musculoskeletal injuries as well as reduce the massive costs placed on our healthcare industry.
\end{abstract}

Keywords: Orthotics; Biomechanics; FHHD; Digital x-rays; Load bearing; Flawed pattern

\section{History}

Orthopedic surgeons, chiropractors, physical therapists and other healthcare specialists have been treating musculoskeletal disorders and the degenerative effects of these disorders the same way for the past four decades. They all steer their entire approach and thinking process towards the reactive model. They assume the patient in front of them is there as a result of some accident, fall or sprain. And while this is the caseat times, they fail to look at the "why" and spend all of their time on the "what". The authors of this study have positioned themselves to claim that biomechanical faults, even when asymptomatic, are far more common than the average healthcare worker realizes. These are the very faults that are the precursors to many injuries as well as premature degenerative changes. In this study, we demonstrate the value of digital $x$-ray for the understanding of underlying musculoskeletal faults on all patients. These faults originate in the foundation of the patient, their feet. The biomechanical collapses found in the 
feet, when corrected with custom orthotics, will then allow the examiner to have a clearer picture of the actual leg length of the patient to determine if there is an anatomical difference, and if so, how much. When the feet are supported with custom orthotics, and femoral head height balance is achieved, with or without the addition of a lift, a more balanced loading of the body will reduce injuries and delay the onset of degenerative changes in the musculoskeletal system.

\section{Introduction}

The total cost of musculoskeletal injuries in the US is undoubtedly huge and the expenses do not limit themselves to the money spent on treatment. The annual expected cost due to musculoskeletal chronic pain was estimated in USD $\$ 1,387,200,000$. (1.387 billion), equivalent to $0.417 \%$ of the national GDP. Lower back pain and osteoarthritis of the knee explained the larger proportion of the total cost, $31.8 \%$ and $27.1 \%$, respectively. Depression attributed to chronic pain is another important consequence accounting for USD \$94 million (Bayesian credibility interval 95\% \$49.1-\$156.26). Productivity losses were also an important cost, although early retirement and presenteeism data are not available (Vargas et al., 2018). The societal impact of musculoskeletal injuries and disorders is not only incalculable but enormous as a burden as well.

Someone would expect that a great amount of effort and resources would be targeted towards the prevention of these injuries from happening in the first place. Knowing that the most popular lifestyle - at least in large urban areas - depends on prolonged sitting, doubtful quality of food and limited physical activity, it seems that related healthcare professionals would choose to focus on why certain injuries occur or become chronic and why the pain persists at times. And although a great amount of effort is already spent in prompting especially the young and productive individuals to obtain a more active and healthy way of life, this does not seem to have led to fewer injuries -rather more.

In the first part of our three-section study, we presented our proactive approach which tries to find the very root of the problem. We were also able to identify five distinguished biomechanically flawed patterns, into which each individual falls if properly tested and scanned. And we truly believe that, if instead of intervening after the injury occurs, we step in before that and correct these imbalances, most musculoskeletal injuries will not happen in the first place. Our approach depends a great deal on measuring the femoral head height difference (fhhd) of a patient. Most authors and researchers agree that fhhd should be less than $3 \mathrm{~mm}$. If a leg length difference greater than $3 \mathrm{~mm}$ is present on digital $\mathrm{x}$-ray, we will make note that the patient is a good candidate for ordering an orthotic device (Corechiropractic, 2020). Others claim that pain and disorders occur with fhhd greater than $5 \mathrm{~mm}$. We might consider that $5 \mathrm{~mm}$ is significant for pain and those individuals with a leg length discrepancy of $5 \mathrm{~mm}$ or greater are more likely to develop low back pain. A leg length discrepancy greater than $10 \mathrm{~mm}$ might predispose that individual to degenerative joint disease (DJD) secondary to biomechanical malposition (Lommel, 2020). To sum up, most researchers agree that those with fhhd < $3 \mathrm{~mm}$ form a "normal" group and those with fhhd $>3 \mathrm{~mm}$ are part of the "abnormal" group of patients.

\section{Materials and Methods}

As stated, in our first study we identified five different flawed patterns and claimed that each human being falls into one of these five categories. Now, in our second study, we try to focus on leg length inequalities as expressed via the measurement of femoral head height difference (fhhd). The bar is set at $3 \mathrm{~mm}$ and that means that any measurement below that is within the normal range and anything above that is abnormal, at least from a biomechanical point of view. Study participants were patients seeking care in our office over a 3-year period. These patient's interests ranged from wanting to learn of their biomechanical imbalances and had little to no symptoms to patients with specific musculoskeletal injuries either from degenerative changes, repetitive motion activity, trauma or a combination of these three. No one was acutely injured or presenting with antalgia or a compensatory posture. All patients presented in their normal postures. There were 351 participants over the 3-year period. All subjects were patients who presented for many different reasons. The youngest patient was 10 while the oldest patient was $79.55 \%$ (193) were male and 45\% (158) were female. 33\% (116) patients ranged between 10-19 years of age.

The test that was performed on these patients was The Maggs' Leg Length Test, which consists of 3 tests. First, a digital laser foot scan of the patient standing in their normal postural position with shoes off is made. The second test consists of a standing A-P L-S digital X-ray of the patient barefoot [3]. And the third test is the same A-P L-S digital x-ray approximately one week later after the patient has inserted their custom foot orthotics into their shoes. The Maggs' Leg Length Test is part of the broader Structural Fingerprint ${ }^{\circledR}$ Exam which is a leading full biomechanical examination of a patient. This examination consists of a consultation, physical examination, digital laser foot scan and 2 standing $\mathrm{x}$-rays of the low back (A-P and lateral) and 2 standing $\mathrm{x}$-rays of the neck (apom and lateral). These are all done barefoot.

Here is some technical information about the Kiosk scanner.
I. About 6 feet tall
II. About 2.5 feet wide
III. About 3 feet deep
IV. Takes 26 key measurements
V. 250 micrometers scan resolution

VI. Uses a Class 2M Diode Laser Product with line generating optics

Our 3D scanners use a laser triangulation method to capture true 3D data. It is accurate to within 250 microns, which is one quarter of a millimeter. By capturing the foot with this much 


\section{Orthopedics and Rheumatology Open Access Journal (OROAJ)}

precision, we can determine imbalances in the plantar vault of one foot to the other. Not only does this provide detailed information for prescribing doctors to discuss with patients but it also gives our lab an accurate depiction of the patient's pedal foundation for crafting custom orthotics (Figure 1).

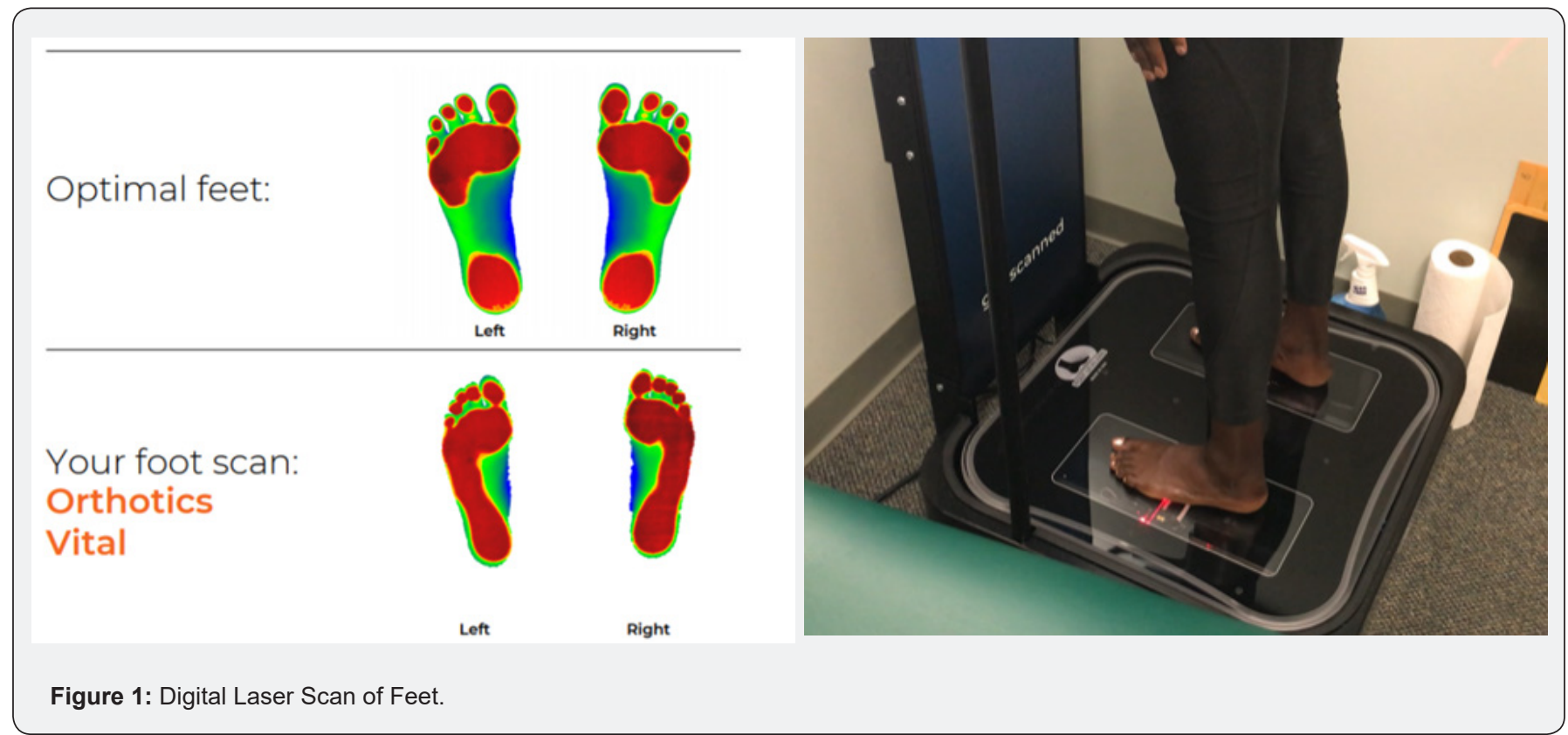

The 3D scanner uses a moving laser to capture the entire plantar surface of the foot, developing a complete topography of the plantar vault. Colorized images are created with red pixels being closest to the laser and blue pixels being farther away. A second pass with a high-definition camera takes a scan of the foot to provide a flesh tone image as well. This allows the provider and our lab to look for sores, lesions, or other foot-related pathology. The interpretation of the digital laser foot scan is as follows; the feet have 3 supportive arches-the lateral arch, the transverse arch and the medial arch. The optimal foot scan shows the foot contacting the ground in the forefoot and heel and is represented in red, with the middle portion of the foot which is not intended to make direct contact with the ground represented in blue. Should any portion of the mid-foot, regardless of which arch it is, present as red, meaning it is making direct contact with the ground, then custom orthotics are ordered, and this patient becomes a viable candidate for this study. All 351 subjects in this study had digital laser foot scans with mild, moderate or severe arch collapse on one or both feet.

When setting patients up for their first A-P L-S x-ray while barefoot, there were several criteria we felt were important. First, many people have internal or external rotation of a foot while standing in their neutral position. Most often this is an adaptation over time to an anatomical leg length difference. We would encourage the patient to stand in the position that was natural for them. Correcting the unilateral rotation could have been recommended to create a symmetrical alignment of the feet and leg, but we elected to have the patient stand in the position that was appropriate for them. Either instruction could be argued as appropriate, but for the sake of the study and to maintain consistency, we elected to have every patient stand in the position they felt most comfortable in.

Secondly, when positioning the patient to take the A-P L-S $\mathrm{x}$-ray, either with shoes on or barefoot, the central ray was 1 " below the umbilicus at 40 " from the tube. The normal central ray is the umbilicus, but 1 " below the umbilicus reduced magnification or distortion of the femoral heads to make measurements more accurate. Furthermore, when re-x-raying the patient with orthotics in their shoes, it was imperative the patient wore new or newer shoes. There was no wear on the heel and if they were tie shoes, we made sure the patient tied them appropriately, so the shoes had minimal effect. The shoe merely held the orthotic in place. When it came to the custom orthotic itself, the orthotic we used to be very important. The orthotic we used to be a relatively thin orthotic, so we would have the patient remove the manufacturer's insert and put our orthotic into their shoe. If for any reason our orthotic was longer than the insert, we instructed the patient on how to trim it so it would fit appropriately. Secondly, our orthotic is a flexible orthotic with shock absorbing materials. It is supportive in all 3 arches of the feet and puts the feet into the correct biomechanical position. We instruct the patient that it generally takes 3-7 days to get used to the orthotic in their shoe. We elected to re-take our $2^{\text {nd }} \mathrm{x}$-ray immediately after the orthotics were placed into the shoes for logistical reasons. It would have presented a scheduling challenge to have the patient come back a week, month or several months later. So, we chose, for consistency purposes, to re-take the x-ray immediately. When measuring the femoral head height, our digital x-ray system comes with built in software that allows 
this measurement to be made very quickly and very accurately. Exclusion criteria included any patient who was unable to stand in normal posture, who didn't want to be x-rayed, who didn't want custom orthotics or who was an amputee.

\section{Results}

In our first study we managed to show that every human being presents with collapsed arches of the foot of some degree, which leads to significant biomechanical and structural alterations of the whole body. These alterations were classified, and five different patterns were identified as a result. We believe that every individual falls into one of the five distinctive flawed patterns that we choose to call Crooked Man (1 through 5) (Figure 2). Now, in this study, our goal is to determine what percentage of the population falls into the "normal" category of fhhd, which is $3 \mathrm{~mm}$ and below, and what percentage falls into the "abnormal" category, which is greater than $3 \mathrm{~mm}$. We had 351 patients in total. We made two $\mathrm{x}$-rays, one with the patient barefoot ( $\mathrm{xr} 1$ ) and one with the patient wearing the custom orthotics that was appropriate for him/her (xr2). Results were as follows:

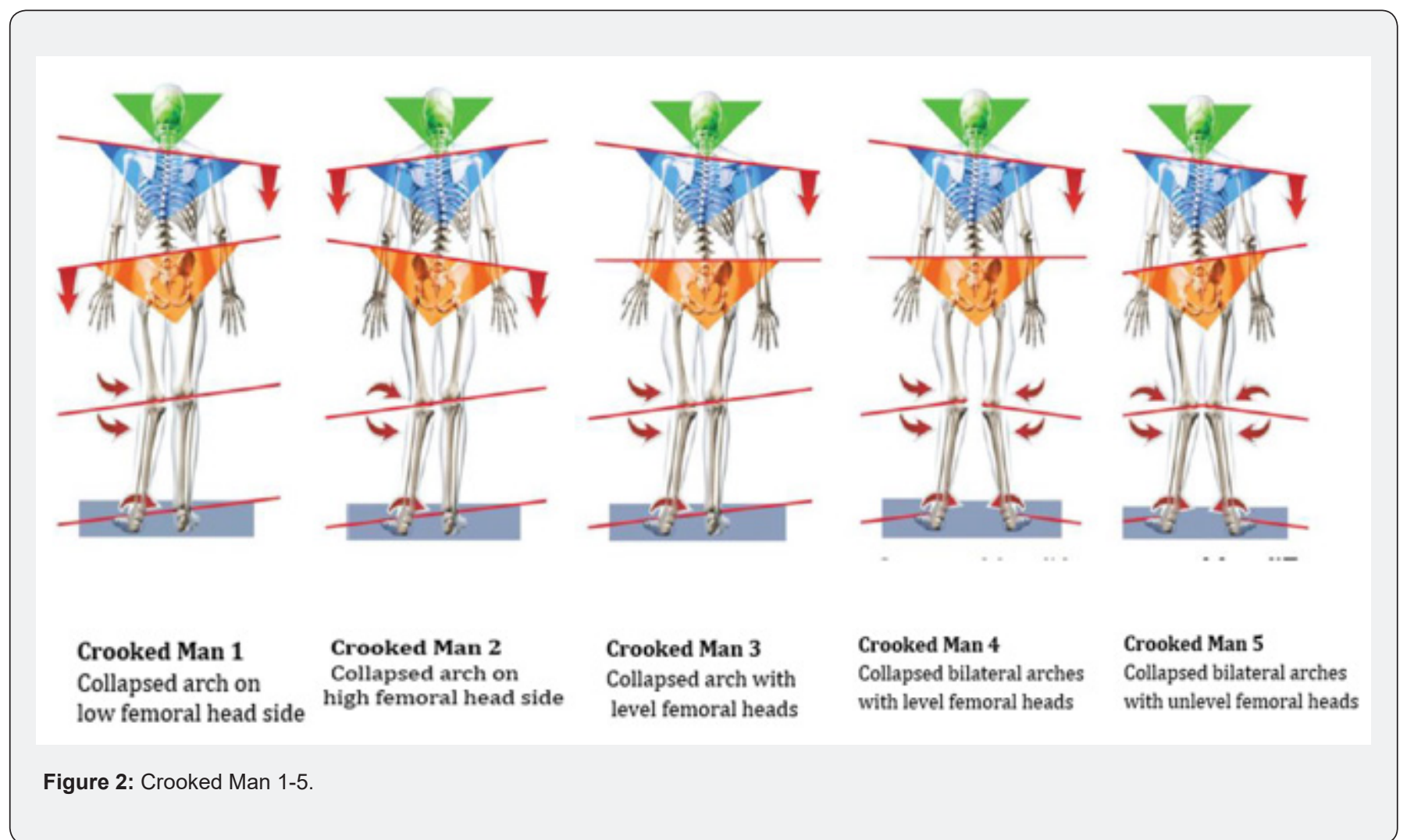

\section{Femoral Head Height Difference}

Definition Criteria: $<3 \mathrm{~mm}$ fhhd = normal, no lift needed

$<1 \mathrm{~mm}$ fhhd from xr1 to $\mathrm{xr} 2=$ no change

$>3 \mathrm{~mm}$ fhhd $=$ abnormal, lift and further evaluation needed

Outcome:

Crooked Man 1: 42\%

Crooked Man 2: 16\%

Crooked Man 3: 6\%

Crooked Man 4: 16\%

Crooked Man 5: 20\%

Without orthotics: $>3 \mathrm{~mm}-224 / 351=64 \%$
$>5 \mathrm{~mm}-147 / 351=42 \%$

With orthotics: $>3 \mathrm{~mm}-214 / 351=61 \%$

$>5 \mathrm{~mm}-141 / 351=40 \%$

Reduced fhhd with orthotics: $147 / 351=42 \%$

Increased fhhd with orthotics: $76 / 351=22 \%$

No change in fhhd with orthotics: $127 / 351=36 \%$

\section{Discussion}

It is well established that the socioeconomic burden of the musculoskeletal diseases in the US is remarkably enormous [4]. Musculoskeletal diagnoses accounted for $18 \%$, or 223.6 million, of the 1.3 billion medical diagnoses, included in hospital discharge records, emergency department and outpatient clinic 
visits, and physician office visits in the United States in 2010 and 2011 (BMUS, 2016) and it is quite safe to assume that nothing has dramatically improved since then - if not worsened. It is also safe to assume that only a handful of healthcare professionals, who examine patients with acute or chronic injuries, devote time and energy to the biomechanical factor. However, our everyday routine with our patients and the final treatment of severe and "unsolved" cases has allowed us to believe that this biomechanical factor actually plays a key role in these injuries' incidence. All we had to do is think backwards and try to prove that if we see the whole body as a biomechanical structure (or a "living machine") and correct these imbalances, then fewer and fewer injuries will occur. And we think we did!

The first most important outcome of our study was that every one of us will eventually fall into one of the five distinct biomechanically flawed patterns that we designed. This is the case since one or both our feet almost always have at least one collapsed arch. And since our feet are the first to bear the ground reaction forces and serve as the foundation of our structure, the rest of the body (knee joints, hip joints, back and pelvis, shoulder joints) will try to adapt to these unevenly distributed loads. These adaptations are highlighted in the Crooked Man (1 to 5) theory.

The second most important outcome and the primary focus of the current study is the fact that, even with orthotic devices on, $61 \%$ of the patients have fhhd $>3 \mathrm{~mm}$ and are, therefore, part of the "abnormal" group and that $40 \%$ have fhhd $>5 \mathrm{~mm}$, which is quite high. Of course, little agreement exists so far regarding the prevalence of limb length inequality, the degree of limb length inequality that is considered clinically significant, and the reliability and validity of assessment methods [5]. Still there are a lot of references which support our claim that fhhd $>3 \mathrm{~mm}$ should not be normal and that fhhd $>5 \mathrm{~mm}$ counts as a moderate case of leg length discrepancy. Subotnick suggests that a limb length inequality greater than $3 \mathrm{~mm}$ may require intervention for runners [6]. Friberg has used $5 \mathrm{~mm}$ of limb length discrepancy as an operational definition for the condition [7]. Holmes has considered $6 \mathrm{~mm}$ of limb length discrepancy as a clinically significant magnitude [8].

With all that being stated, our results become even more meaningful. Six out of 10 individuals were found with fhhd greater than $3 \mathrm{~mm}$ and 4 out of 10 had fhhd greater than $5 \mathrm{~mm}$ with orthotics on! Given the fact that orthotics should always be prescribed when collapsed arches of the foot are present, these results can only mean that orthotics are just not enough to achieve optimal function of the body and that further biomechanical evaluation is warranted. The latter is $100 \%$ consistent with our approach which is based on a full load-bearing assessment of every individual in order not only to treat but to prevent injuries from happening, which is of course what this study is all about in the first place. To summarize, what we did is that we tried to integrate biomechanical, digitally obtained x-rays of each patient in our current approach. Our results far exceed the statistically significant values and show how many parameters are involved when we try to optimize the function of the musculoskeletal system. These parameters are seriously overlooked, however, with a simple x-ray scan of the patient we can make an important step towards the goal which is injury prevention.

\section{Conclusion}

Limited action has been taken so far by the healthcare community regarding musculoskeletal injury prevention protocols. Instead, it seems that more and more resources are directed towards the treatment of both acute and chronic injuries, something that is illustrated in the US socioeconomic burden data annually. Our study, based on current literature, accurate measurements and on our clinical experience suggests a different, more proactive approach to tackle this type of ailments. We need to start seeing the human body as a whole and as a living and running machine. Using the biomechanical factor as a spearhead, we will be able to walk a path towards less injuries.

\section{References}

1. Constanza Vargas, Norberto Bilbeny, Carlos Balmaceda, María Francisca Rodríguez, Pedro Zitko, et al. (2018) Costs and consequences of chronic pain due to musculoskeletal disorders from a health system perspective in Chile. Pain Rep 3(5): e656.

2. Leg Length Discrepancy: The Overlooked Reason Why Your Neck and Back Hurt (2020).

3. Inside Spinal Pelvic Stabilizer Research - Standard Protocols for Treating Leg Length Inequality.

4. The Burden of Musculoskeletal Diseases in the United States Prevalence, Societal and Economic Cost 2016.

5. Rebecca J Brady, John B Dean, T Marc Skinner, Michael T Gross (2003) Limb Length Inequality: Clinical Implications for Assessment and Intervention. Journal of Orthopaedic \& Sports Physical Therap pp. 221-234.

6. S I Subotnick (1981) Limb length discrepancies of the lower extremity (the short leg syndrome) by S. Subotnick J Orthop Sports Phys Ther 3(1): 11-16

7. Friberg O, Nurminen M, Korhonen K, Soininen E, Manttari T (1988) Accuracy and precision of clinical estimation of leg length inequality and lumbar scoliosis: comparison of clinical and radiological measurements. Int Disabil Stud 10(2): 49-53.

8. J C Holmes, A L Pruitt, N J Whalen (1993) Iliotibial band syndrome in cyclists by Holmes JC, Pruitt AL, Whalen NJ. Am J Sports Med 21(3): 419-424. 
CC (i) This work is licensed under Creative Commons Attribution 4.0 License

DOI: 10.19080/OROAJ.2021.17.555970

\section{Your next submission with Juniper Publishers} will reach you the below assets

- Quality Editorial service

- Swift Peer Review

- Reprints availability

- E-prints Service

- Manuscript Podcast for convenient understanding

- Global attainment for your research

- Manuscript accessibility in different formats

( Pdf, E-pub, Full Text, Audio)

- Unceasing customer service

Track the below URL for one-step submission https://juniperpublishers.com/online-submission.php 